\title{
Impact of capacitance and tunneling asymmetries on Coulomb blockade edges and Kondo peaks in nonequilibrium transport through molecular quantum dots
}

\author{
A. A. Aligia \\ Centro Atómico Bariloche and Instituto Balseiro, Comisión Nacional de Energía Atómica, 8400 Bariloche, Argentina \\ P. Roura-Bas \\ Departamento de Física, Centro Atómico Constituyentes, Comisión Nacional de Energía Atómica, 1650 Buenos Aires, Argentina \\ Serge Florens \\ Institut Néel, CNRS et UJF, BP 166, 38042 Grenoble Cedex 9, France \\ (Received 14 May 2015; revised manuscript received 17 June 2015; published 6 July 2015)
}

\begin{abstract}
We investigate theoretically the nonequilibrium transport through a molecular quantum dot as a function of gate and bias voltage, taking into account the typical situation in molecular electronics. In this respect, our study includes asymmetries both in the capacitances and tunneling rates to the source and drain electrodes, as well as an infinitely large charging energy on the molecule. Our calculations are based on the out-of-equilibrium noncrossing approximation (NCA), which is a reliable technique in the regime under consideration. We find that Coulomb blockade edges and Kondo peaks display strong renormalization in their width and intensity as a function of these asymmetries, and that basic expectations from Coulomb blockade theory must be taken with care in general, especially when Kondo physics is at play. In order to help comparison of theory to experiments, we also propose a simple phenomenological model which reproduces semiquantitatively the Coulomb blockade edges that were numerically computed from the NCA in all regimes of parameters.
\end{abstract}

DOI: 10.1103/PhysRevB.92.035404

PACS number(s): 73.63.-b, 73.63.Kv, 72.15.Qm

\section{INTRODUCTION}

In the last decade, an enormous amount of research in the field of nanoelectronics has been devoted to the study of electronic transport through quantum dots. A seminal and very versatile type of device is the gate-defined artificial atom created at the two-dimensional electron gas confined in semiconducting heterostructures [1-4]. More recently, molecular quantum dots [5-12] have gained an important momentum, due to the possibility of playing with the chemistry of the molecule, which compensates the reduced gate tunability of these systems. In all these quantum dot setups, several physical effects are generically observed when the system is cooled at cryogenic temperatures: Coulomb blockade is probably the major one, due to the large charging effects in these nanoscopic quantum dots. In addition, more subtle correlations show up due to the Kondo effect [13,14], which implies a resonance at the Fermi energy in the spectral density of the dot state, which in turn leads to an anomalous peak in the differential conductance $G(V)=d I / d V$ at zero bias voltage $V$.

However, important and unexpected correlation effects can also take place in transport features at finite bias voltage. For instance, Coulomb blockade edges were shown by Könemann et al. [15] to present a strong width renormalization in the situation of large tunnel asymmetries between source and drain electrodes, that is also quite typical in molecular quantum dots [16]. Enhancement of cotunneling features of excitation levels (as a precursor of the Kondo effect) were also described by Paaske et al. [17] and later widely observed. All these features need to be incorporated in the current theoretical developments of nonequilibrium quantum transport, which has so far mainly focused on analyzing the main universal Kondo signatures in $G(V)$ under many simplifying assumptions (such as symmetric capacitive and tunnel coupling to the electrodes). In particular, the experiment by Könemann et al. [15] investigated a strongly confined semiconducting quantum dot coupled to two conducting leads with highly asymmetric tunnel couplings, and studied tunneling resonances at nearly opposite values of $V$. While in the absence of interaction effects one expects nearly the same intensity and width of the two resonances (see Sec. III), the Coulomb blockade edge at positive voltage was however nearly five times more intense and nearly two times narrower than the peak at negative voltage. The authors assumed that for negative voltage, the width in the spectral density of the dot level is two times larger than for positive voltage. This is based on the fact that the charge-transfer peak (the one near the dot level $E_{d}$ ) in the density of states of the Anderson model is two times wider when the dot is occupied $[18,19]$. However, as we shall show, the peak for negative voltage corresponds to the intermediate-valence (IV) regime, in which the width of the spectral density is approximately $3 / 2$ times larger than in the empty-orbital regime. In addition, the ratio of widths for negative and positive voltages depends also on the asymmetries of the source and drain capacitances. Our calculation will also be able to take into account the presence of a Kondo resonance at small bias, which in previous Coulomb blockade theory was clearly lacking [15].

In this paper, after a brief discussion of what is expected in the absence of Coulomb interactions for asymmetric coupling to the leads, we calculate the differential conductance $d I / d V$ in the Keldysh formalism within the noncrossing approximation (NCA) [20,21]. The NCA reproduces well the scaling relations with temperature $T$ and bias voltage in the Kondo regime [22] and was for example also successfully used to interpret experimental results on a controlled crossover between SU(4) and SU(2) Kondo states driven by magnetic 
field in a nanoscale Si transistor [23], as well as quantum phase transitions involving singlet and triplet states in molecular quantum dots [9]. It was also used recently to discuss nonequilibrium Kondo spectroscopy in a system of double quantum dots [24]. For asymmetric coupling to the leads, we will also derive an analytical expression which describes semiquantitatively the NCA results. This expression is used to interpret the experiments of Könemann et al. [15] providing a good agreement, and could be used for further studies of molecular systems.

The paper is organized as follows. In Sec. II we explain our main assumptions, and present the Hamiltonian and the equation describing the current in the presence of a finite bias. In Sec. III we compute for comparison the differential conductance $G(V)$ in the absence of Coulomb interaction. Section IV contains the NCA results for the nonlinear conductance, and presents an analysis of the positions and widths of the peaks displayed in $G(V)$ for different experimentally relevant conditions. In Sec. V we discuss the experiment of Könemann et al., and end up in Sec. VI with a summary.

\section{MODEL AND ASSUMPTIONS}

We consider either a single molecule or a small semiconducting quantum dot coupled to two conducting leads. For molecules or small dots, the separation between the levels $\delta$ is much larger than the coupling to the leads $\Delta_{v}$ defined below and we can include a single localized level of the dot in the model. This reduces the heating effects always present in small devices [25-27], because inelastic cotunneling processes involving excitons in the dot [25,26] are inhibited. In fact, for example comparison between theory and experiment for a spin-1 Kondo effect in a molecule indicates that an equilibrium temperature is well established at very low temperatures [7]. However, the regime of large voltage drop is in general more difficult to address, and important heating could indeed take place. Including phonons and thermalization effects is still an open problem in the presence of Kondo correlations to the best of our knowledge. Nevertheless, our study has mainly focused on regimes where the molecule is in strong contact with one of the two leads, which makes it in a good equilibrium.

We neglect here the effects of phonons which are important in large grains [26] and in molecules [28-31]. These effects can be incorporated in our NCA formalism [32], and their main manifestations are a narrowing of the peaks due to renormalization of the hybridization and the Kondo temperature, and the appearance of small replicas of the peaks in the conductance discussed here shifted at multiples of the phonon frequencies.

We therefore consider the one-level Anderson impurity model with infinite on-site repulsion $U$, an assumption that is realistic for most molecular quantum dots, but also well obeyed for very small semiconducting devices in the vicinity of a charge-degeneracy point. The impurity states thus involve a singlet configuration $|s\rangle$ together with a spin doublet $|\sigma\rangle$ ( $\sigma=\uparrow$ or $\downarrow$ ) corresponding to one additional electron (or hole) in the dot, taking this particle from the left $(L)$ or right $(R)$ conducting leads to which the dot is connected. The
Hamiltonian thus reads

$$
\begin{aligned}
H= & \sum_{\sigma} E_{d}|\sigma\rangle\langle\sigma|+\sum_{\nu k \sigma} \epsilon_{\nu k} c_{\nu k \sigma}^{\dagger} c_{\nu k \sigma} \\
& +\sum_{\nu k \sigma}\left(V_{k}^{v}|\sigma\rangle\langle s| c_{\nu k \sigma}+\text { H.c. }\right),
\end{aligned}
$$

where the constraint $|s\rangle\left\langle s\left|+\sum_{\sigma}\right| \sigma\right\rangle\langle\sigma|=1$ is imposed. Here $c_{v k \sigma}^{\dagger}$ create conduction states in the lead $v$ with spin $\sigma$ and wave vector $k$. The tunnel couplings of the quantum dot to the leads are $\Delta_{v}=\pi \sum_{k}\left|V_{k}^{v}\right|^{2} \delta\left(\omega-\epsilon_{v k}\right)$, assumed independent of energy $\omega$, and we define the total hybridization $\Delta=\Delta_{L}+\Delta_{R}$. We take the sign of the bias voltage in such a way that $\mu_{L}-\mu_{R}=e V$, where $\mu_{v}$ is the chemical potential of lead $\nu$. Note that the interchange of right and left leads or electrons by holes is equivalent to a change of sign of $V$. We take the arbitrary origin of energies at $\mu_{R}=0$. The capacitance effects modify the energy necessary to add an electron to the dot with the lever arm parameter $\alpha$ (which depends on the source, drain, and gate capacitances) $[15,16]$, defined as

$$
E_{d}=E_{d}^{0}+\alpha e V
$$

where $0 \leqslant \alpha \leqslant 1$, and $e$ is the electron charge (taken positive). For the situation of symmetric voltage drop that is usually considered, $\alpha$ is often taken near $1 / 2$, but this may not be the case in many experimental setups.

We obtain the conductance $G=d I / d V$ differentiating the current $I$ (numerically from NCA results), which is given by [33]

$$
\begin{aligned}
I & =C \int d \omega \rho\left(\omega, V, E_{d}\right)\left[f_{L}(\omega)-f_{R}(\omega)\right], \\
C & =\frac{8 \pi e \Delta_{L} \Delta_{R}}{h \Delta},
\end{aligned}
$$

where $f_{v}(\omega)=f\left(\omega-\mu_{v}\right)$ is the Fermi distribution in each lead, with $f(\omega)=1 /\left(e^{\omega / k T}+1\right)$, and $\rho\left(\omega, V, E_{d}\right)$ is the nonequilibrium (voltage-dependent) spectral density of the impurity level.

\section{NONINTERACTING RESONANT-LEVEL MODEL}

Before discussing in more detail the complex effects of Coulomb interactions, we present in this section a simplified model that sheds light onto the expected behavior of the differential conductance $G(V)$. The first assumption that we make in this section is that the tunnel couplings to the leads are very asymmetric. This hypothesis is realistic for molecular quantum dots and also in the experiment of Könemann et al. in Ref. [15]. We make here the choice $\Delta_{R} \gg \Delta_{L}$, so that the system is in equilibrium with the right lead and the explicit dependence on the voltage of the spectral density $\rho(\omega)$ disappears [the dependence of $\rho(\omega)$ with $V$ through $E_{d}$ given 
by Eq. (2) remains]. Then one has

$$
\rho\left(\omega, V, E_{d}\right) \simeq \rho_{R}\left(\omega, E_{d}\right),
$$

where $\rho_{R}\left(\omega, E_{d}\right)$ is the spectral density of the impurity states in equilibrium with the right lead, for a dot energy given by Eq. (2). Using Eqs. (2)-(4), one obtains at $T=0$

$$
\begin{aligned}
G(V)= & C e \rho_{R}\left(e V, E_{d}^{0}+\alpha e V\right) \\
& +\alpha C e \int_{0}^{e V} d \omega \frac{\partial \rho_{R}\left(\omega, E_{d}^{0}+\alpha e V\right)}{\partial E_{d}} .
\end{aligned}
$$

In the limit of asymmetric capacitances $\alpha \rightarrow 0$, only the first term survives and $G(V)$ is just a map of the spectral density $\rho_{R}\left(\omega, E_{d}^{0}\right)$, as is usual with experiments with a scanning tunneling microscope (STM). Therefore, in this limit, $G(V)$ has a single peak located at $V=E_{d}^{0} / e$ (the charge-transfer peak), and no peak for the opposite bias voltage. In addition, a Kondo peak at $V=0$ can show up in the presence of Coulomb interactions, if $-E_{d}^{0} \gg \Delta$.

The main assumption that we make in this section is to take a noninteracting resonant level spectral density of width $2 \Delta$ at half maximum, $\rho_{R}\left(\omega, E_{d}\right) \simeq \rho_{0}\left(\omega-E_{d}\right)$, with

$$
\rho_{0}\left(\omega-E_{d}\right)=\frac{\Delta / \pi}{\left(\omega-E_{d}\right)^{2}+\Delta^{2}} .
$$

This is a crude approximation which is certainly not valid in the presence of interactions (it misses the important Kondo anomaly), but it can describe qualitatively the position and width of the Coulomb peaks in $G(V)$ derived from the charge-transfer peak in the spectral density of the dot states, which can be renormalized from bare values by the interaction, as we discuss in Sec. IV B 2. Assuming, as in Eq. (6), that $\partial \rho_{R} / \partial E_{d}=-\partial \rho_{R} / \partial \omega$ (but not $\rho_{R}=\rho_{0}$ yet), the second term of Eq. (5) can be easily evaluated and one obtains

$$
G(V) \simeq C e\left[(1-\alpha) \rho_{R}\left(e V, E_{d}\right)+\alpha \rho_{R}\left(0, E_{d}\right)\right],
$$

noting that $E_{d}$ displays an explicit dependence upon the voltage from Eq. (2). While the assumption made above for the derivatives of the current is not exactly true (even in equilibrium) because of the dependence of the weight and width of the peaks with $E_{d}$, this simple expression reflects a general feature of the expected Coulomb peaks, in connection to the charge-transfer peak of the equilibrium spectral density $\rho_{R}$. Indeed, now $G(V)$ displays two finite bias peaks, one that is similar to the STM case and is located at voltage $e V=E_{d}$ [namely $e V=E_{d}^{0} /(1-\alpha)$ ], and a second "intermediate-valence" (IV) peak at an opposite voltage value such that $E_{d}=E_{d}^{0}+\alpha e V=0$. In this latter case, the impurity experiences indeed maximal charge fluctuations because the level is located exactly on-resonance, and thus one can expect a different width renormalization than for the STM peak, due to the different influence of the Coulomb interaction, as we will demonstrate later on. For positive $E_{d}^{0}$ (corresponding to a vanishing charge in the dot, also called the empty orbital regime), the STM peak lies at positive bias and the IV peak is located at negative bias, and conversely for negative $E_{d}^{0}$ (corresponding to a spin-degenerate odd charge in the dot, also called the local-moment regime). Thus, if $\rho_{R}=\rho_{0}$ is assumed (which is valid only in the absence of Coulomb interaction), the conductance $G(V)$ displays two peaks as a function of voltage: (i) an "STM-like" peak at $V=E_{d}^{0} /[(1-\alpha) e]$ of width $2 \Delta /[(1-\alpha) e]$ and maximum intensity $(1-\alpha) e C / \Delta$; (ii) an IV peak at $V=-E_{d}^{0} /(\alpha e)$ of width $2 \Delta /(\alpha e)$ and maximum intensity $\alpha e C / \Delta$. Therefore, in the absence of Coulomb interactions, the amplitude and width renormalizations are related, and cannot reproduce the experimental observations made in Ref. [15]. We now turn to a full microscopic calculation of the problem, without recourse to the many assumptions made above, that incorporates the correct nature of the Coulomb blockade edges and Kondo resonances for all parameters in the device.

\section{NCA RESULTS FOR THE INTERACTING MODEL}

\section{A. The equilibrium spectral density}

We consider now Hamiltonian (1) in its full complexity for the case of a finite bias voltage. The noncrossing approximation (NCA) has proved in the past an excellent technique to deal with large Coulomb interactions, and can be extended to the present nonequilibrium situation. In order to gain intuition, we first focus on the zero bias $(V=0)$ spectral density of the localized level, which is shown on Fig. 1 for low temperatures and three values of the dot energy level $E_{d}$, corresponding to the empty-orbital $\left(E_{d}=4 \Delta\right)$, local-moment $\left(E_{d}=-4 \Delta\right)$, and mixed-valence $\left(E_{d} \sim 0\right)$ regimes [34]. For positive $E_{d}$ the result is very similar to the noninteracting resonant level prediction, Eq. (6), with a spectral width $2 \Delta$, except for the fact that the position of the peak is slightly shifted to higher energy $E_{d}^{\text {eff }}=4.72 \Delta$. This energy shift $E_{d}^{\text {eff }}-E_{d}$ is in very good agreement with the Haldane shift [35] $(\Delta / \pi) \ln (D / \Delta)=0.733 \Delta$ for the half bandwidth $D=10 \Delta$ that we have used in the calculations. This is expected because the NCA incorporates naturally the lowest order perturbative

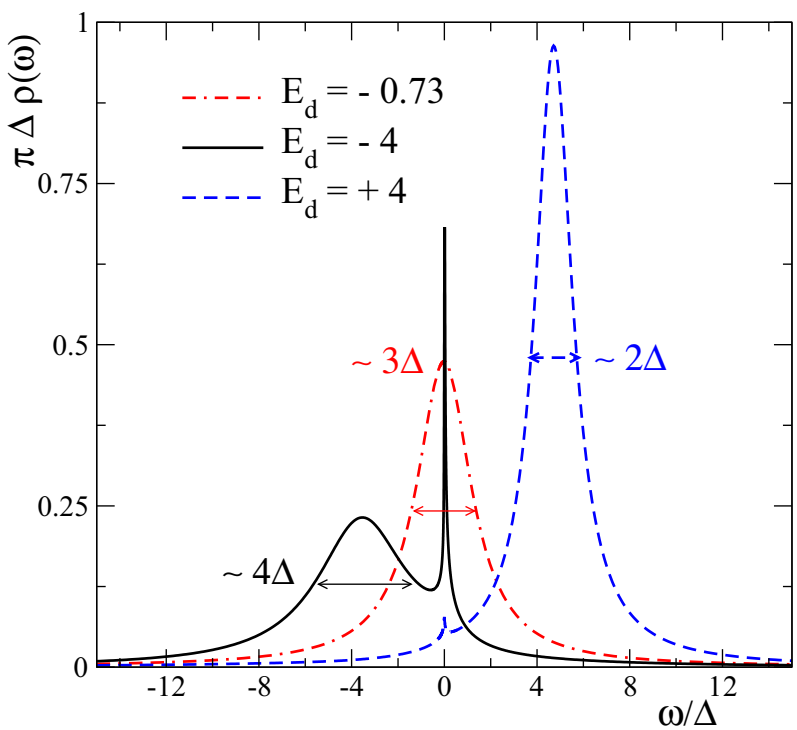

FIG. 1. (Color online) Equilibrium $(V=0)$ spectral density of the localized state for the infinite-interaction Anderson model in the empty-orbital regime (dashed line; $E_{d}=4 \Delta, T=0.01 \Delta$ ), in the intermediate-valence (IV) regime (dash-dotted line; $E_{d}=-0.733 \Delta$, $T=\Delta$ ) [34], and in the local-moment regime (full line; $E_{d}=-4 \Delta$, $T=0.01 \Delta$ ) that shows an additional Kondo feature at low energy. 
terms of the development in the hybridization $V_{k}^{v}$. Note that the small spike at the Fermi energy $\omega=0$ is an artifact of the NCA in the empty-orbital regime.

In the local-moment regime at negative $E_{d}$, the chargetransfer peak is nearly two times wider due to the underlying extra spin degeneracy, as was discussed in the introduction and in previous works $[18,19]$, and the NCA correctly reproduces this behavior. The total intensity is also reduced by correlation effects, as discussed phenomenologically in Sec. IV B 2, and the position of the peak is also shifted to higher energies. More importantly, the screening of the spin-degenerate states leads to a Kondo peak at the Fermi level, a nontrivial effect that the NCA is able to describe properly.

Finally, the density of states for the mixed-valence situation $\left(E_{d}^{\text {eff }}=0\right)$ shows a peak centered at the Fermi level as expected, with a width of about $3 \Delta$ that interpolates between the widths found in the empty-orbital and local-moment regimes. The temperature in this calculation has been increased to avoid a shortcoming of the NCA [34].

Regarding the conductance $G(V)$, these results anticipate similarities and differences with the naive expectations based on the noninteracting resonant level model discussed at the end of the previous section (apart from the obvious lack of Kondo resonance in the latter model). A minor point is that $E_{d}^{0}$ should be replaced by an effective level $E_{d}^{0, \text { eff }}$, which includes the Haldane shift. Based on Fig. 1 and Eq. (7) the width of the STM-like peak in the empty-orbital regime should be $2 \Delta /[(1-\alpha) e]$, thus $4 \Delta$ at $\alpha=1 / 2$ for a symmetric voltage drop (as for the noninteracting resonant level model). However, the width of the STM peak in the local-moment regime should rather be $4 \Delta /[(1-\alpha) e]$, thus $8 \Delta$ at $\alpha=1 / 2$. More subtly, the IV peak (located at opposite voltage to the STM peak) should have the same width for the empty-orbital and local-moment regimes, namely $3 \Delta /[(1-\alpha) e]$, thus $6 \Delta$ at $\alpha=1 / 2$. These general trends, that we will now examine microscopically from full NCA calculations of the conductance, show that the experimental analysis of the width of Coulomb blockade peak must be done with care.

\section{B. Conductance for asymmetric tunnel coupling to the leads}

\section{Numerical NCA results}

In this subsection, we calculate the conductance using the nonequilibrium formalism within the noncrossing approximation (NCA) $[20,21]$ for a large choice of parameters. Details on the technique are given for example in Ref. [36]. We choose here asymmetric tunnel coupling to the leads $\Delta_{R}=60 \Delta_{L}$, and set the reference energy scale as $\Delta=\Delta_{L}+\Delta_{R}=1$, with a temperature $T=0.01$ and a half bandwidth of the leads $D=10$. Owing to the large ratio $\Delta_{R} / \Delta_{L} \gg 1$, the impurity is effectively in equilibrium with the right lead, although the calculations were in practice performed using the full out-of-equilibrium NCA.

In Fig. 2 we show $G(V)$ in units of the conductance quantum $G_{0}=2 e^{2} / h$ for positive $E_{d}^{0}$ (empty-orbital regime) and several values of capacitance asymmetries $\alpha$. The small spike at $V=0$ is again an artifact of the NCA and should be disregarded. For symmetric voltage drop, $\alpha=1 / 2$, the discussion of Sec. III based on the noninteracting resonant

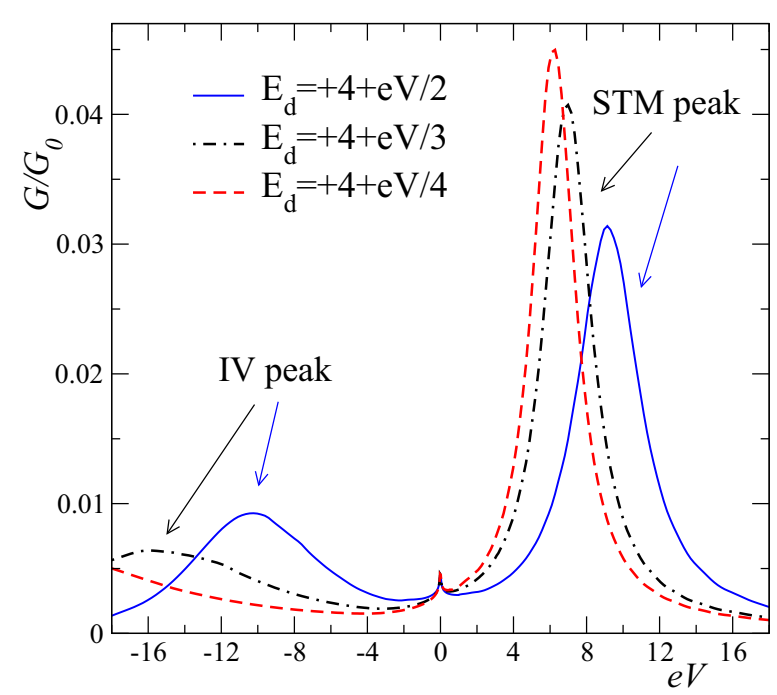

FIG. 2. (Color online) Finite bias differential conductance $G(V)$ in the empty-orbital regime $E_{d}^{0}=4 \Delta=4$ for several values of the capacitance ratio $\alpha=1 / 2,1 / 3,1 / 4$ defined according to Eq. (2), and with a hybridization ratio $\Delta_{R} / \Delta_{L}=60$ (the impurity is here in equilibrium with the right lead).

level predicts two peaks of equal width $4 \Delta$ and equal height $e C /(2 \Delta)$ at $e V= \pm 2 E_{d}^{0, \text { eff }} \approx 9.4$. However, the more refined analysis of Sec. IV A, based on the general features of the interacting spectral density, predicts different width renormalization: a width $4 \Delta$ for the STM peak, and a width $6 \Delta$ for the IV peak. These trends are well described by the full NCA calculation shown in Fig. 2. However, the conductance $G=d I / d V$ is not directly given by the spectral density, but the dependencies of its effective width and weight with $E_{d}$ affect its shape [second term of Eq. (5)]. This is particularly important for the IV peak, for which these dependencies are larger. This will be discussed in more detail in the next subsection. The width of the IV peak within the NCA is about $20 \%$ larger than $6 \Delta$. This can be due to the above mentioned effects or to the limitations of the NCA at low temperatures in the IV regime. In any case, our results demonstrate that the STM and IV peaks are generically asymmetrical, and that their widths depend sensitively on the charge state present on the quantum dot.

As $\alpha$ decreases, the tendencies predicted by the simple analysis of Sec. III remain qualitatively valid. The position of the STM peak tends to $E_{d}^{0}$ and its width to $2 \Delta$ in the limit $\alpha \rightarrow 0$. In contrast, the IV peak broadens enormously and moves to more negative values of $V$ when the capacitances become very asymmetric.

In Fig. 3 we show the results for the local-moment regime at negative $E_{d}^{0}$. In this case, both peaks are much broader than the predictions of the naive expectations based on a noninteracting resonant-level spectral density. Indeed, the STM peak now lying at negative voltage shows strong interaction effects (already witnessed in the spectral density in Fig. 1) that make it twice broader than without interactions (the width is $8 \Delta$ for symmetric voltage drop $\alpha=1 / 2$ ). The IV peak, now located at positive bias voltage, displays again a width that is about $3 / 2$ wider than the simple expectations outlined at the end of 


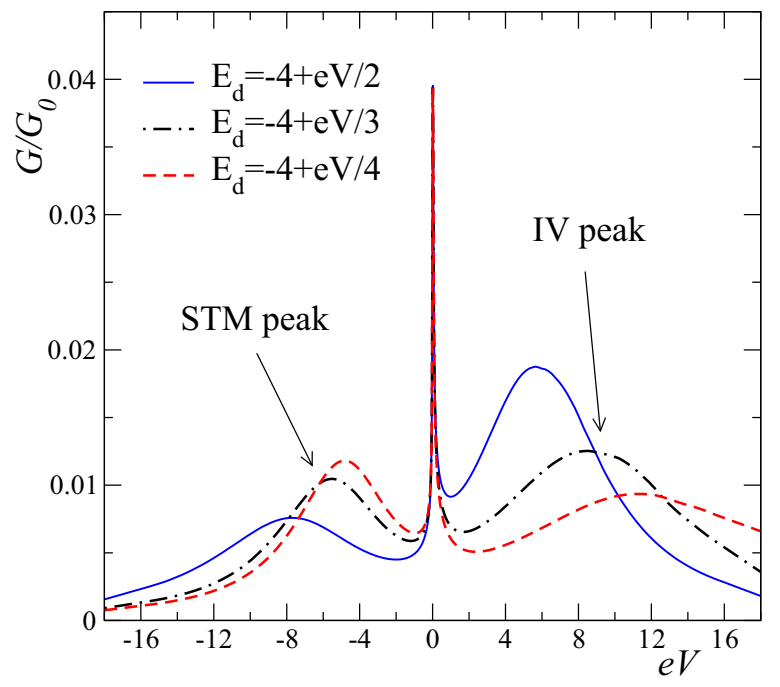

FIG. 3. (Color online) Finite bias differential conductance $G(V)$ in the local-moment regime $E_{d}^{0}=-4 \Delta=-4$ for several values of the capacitance ratio $\alpha=1 / 2,1 / 3,1 / 4$ defined according to Eq. (2), and with a hybridization ratio $\Delta_{R} / \Delta_{L}=60$.

Sec. III, due to combined charge fluctuations and Coulomb effects (the width is $6 \Delta$ for $\alpha=1 / 2$ ). The IV peak is also shifted to the left, since for $\alpha=1 / 2$ one would expect it to lie at $e V=2 E_{d}^{0, \text { eff }} \approx 6.5 \Delta$, while it is located at $5.7 \Delta$. While naively one would expect a ratio of maxima inversely proportional to the width, the intensity of the STM peak is smaller than expected, especially for $\alpha=1 / 2$. This further correlation effect is discussed in the next subsection where we present a more refined analytical approach. Again, as $\alpha$ decreases, the width and intensity of both peaks evolve in agreement with the expectations of the noninteracting resonant-level model.

As expected, the most drastic difference from the noninteracting resonant-level model for negative $E_{d}^{0}$ is the appearance of the Kondo peak at $V=0$. Note that its main features are independent of $\alpha$, which will be discussed in Sec. IV C 2. We now turn to a physical interpretation of the numerical NCA result.

\section{Phenomenological approach}

The main reason for the failure of the noninteracting resonant-level model to account for Coulomb blockade peaks at asymmetric tunnel coupling to the leads is that the correct width and weight of the peak in the impurity level spectral density $\rho(\omega)$ both depend in a nontrivial way on the occupancy, as discussed in Sec. IV A. One of the simplest ways to modelize this effect is the alloy analogy approach to periodic Anderson models, in which the electrons with spin down are frozen to calculate the dynamics of electrons with spin up and vice versa $[37,38]$. This approach is also related to the so-called Hubbard-III approximation [39], in which the weight of the charge-transfer peak near $E_{d}$ for spin $\sigma$ (or the lower Hubbard band in a Hubbard model) is proportional to the number of unoccupied states with the opposite spin, $1-\left\langle n_{-\sigma}\right\rangle$.

To correct the width inconsistency in the noninteracting resonant-level model, we have fitted the width of the NCA peak as a function of $E_{d}$ at a temperature $T=2 \Delta$ (such that the Kondo peak is absent and the width of the IV peak is well behaved [34]), with other parameters taken as in Sec. IV B 1. For simplicity, in this section and in Sec. V, we replace the notation $E_{d}^{\text {eff }}$ by $E_{d}$, incorporating the Haldane shift in the latter. From this, we obtain that the effective half-width at half maximum can be approximated as

$$
\frac{\Delta_{\text {eff }}}{\Delta}=\frac{3}{2}-\frac{1}{2} \tanh \left(\frac{E_{d}}{2.77 \Delta}\right)
$$

This simple formula nicely interpolates between the emptyorbital case $\left(\Delta_{\text {eff }}=\Delta\right.$ for $\left.E_{d} \gg \Delta\right)$ and the local-moment regime $\left(\Delta_{\text {eff }}=2 \Delta\right.$ for $\left.-E_{d} \gg-\Delta\right)$, passing through the mixed-valence regime $\left[\Delta_{\text {eff }}=(3 / 2) \Delta\right.$ for $\left.\left|E_{d}\right| \ll \Delta\right]$.

The above discussion suggests that a better description of the single peak in the equilibrium local density of state can be obtained using the improved formula:

$$
\rho_{R}\left(\omega, E_{d}\right)=\frac{\left(1-\left\langle n_{\sigma}\right\rangle\right) \Delta_{\mathrm{eff}} / \pi}{\left(\omega-E_{d}\right)^{2}+\Delta_{\mathrm{eff}}^{2}},
$$

where $\left\langle n_{\sigma}\right\rangle=\int d \omega \rho_{R}(\omega) f_{R}(\omega)$ is the occupation of the dot for a given spin, independent of the spin in the absence of an applied magnetic field. Integration gives $\left\langle n_{\sigma}\right\rangle$ in terms of the digamma function $\psi(x)$ :

$$
\begin{aligned}
\left\langle n_{\sigma}\right\rangle & =\frac{1 / 2-\Psi_{R}}{3 / 2-\Psi_{R}}, \\
\Psi_{v} & =\frac{1}{\pi} \operatorname{Im} \psi\left[\frac{1}{2}+\frac{\Delta_{\text {eff }}+i\left(E_{d}-\mu_{\nu}\right)}{2 \pi T}\right] .
\end{aligned}
$$

Replacing then Eq. (9) in Eq. (3) gives the current:

$$
I=C\left(1-\left\langle n_{\sigma}\right\rangle\right)\left(\Psi_{R}-\Psi_{L}\right) .
$$

Finally differentiating this expression, one obtains an analytical although lengthy expression for the differential conductance $G(V)$, which we do not reproduce here. At $T=0, G(V)$ contains two terms which have the form of those included in Eq. (7), plus some additional terms due to the dependence of $\Delta_{\text {eff }}$ and $\left\langle n_{d \sigma}\right\rangle$ with respect to $E_{d}$. These additional terms affect the IV peak, for which the variation of $\Delta_{\text {eff }}$ and $\left\langle n_{d \sigma}\right\rangle$ is significant.

In Fig. 4 we compare the conductance $G(V)$ obtained through this phenomenological approach to the full NCA result in the empty orbital case $E_{d}^{0}=4 \Delta$ (note that the Haldane shift is included by hand in the level position within the phenomenological approach). We remind the reader that for $\alpha=1 / 2$, the noninteracting resonant-level model predicts a symmetric $G(V)=G(-V)$ conductance curve, which turns out to be incorrect in the presence of strong Coulomb interaction. The phenomenological approach is thus a considerable improvement for the IV peak at negative voltage, although some quantitative discrepancies remain. In particular, the analytical expression gives an intermediate-valence peak a little bit narrower and shifted towards $V=0$, but the general trends are well reproduced by the simple approach. In addition, this discrepancy might be due to limitations of the NCA at low temperatures in this regime.

In the local-moment regime for negative $E_{d}^{0}$, as shown in Fig. 5, the comparison is less satisfactory. The Kondo peak is obviously missing in the phenomenological approach and the 


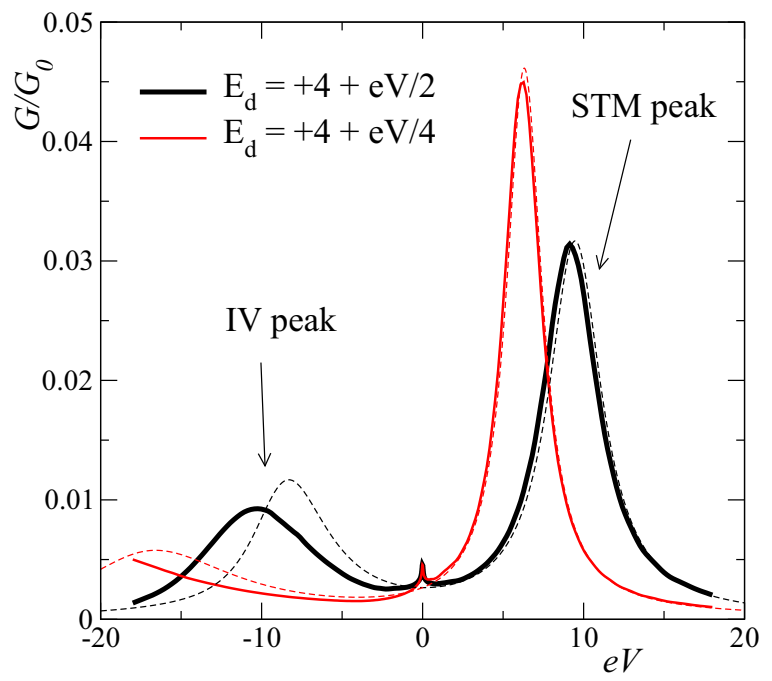

FIG. 4. (Color online) Differential conductance in the emptyorbital regime $E_{d}^{0}=4 \Delta=4$, for asymmetric tunnel couplings $\left(\Delta_{R}=60 \Delta_{L}\right)$, low temperature $T=0.01 \Delta$, and two values of the capacitance ratio $\alpha=1 / 2,1 / 4$. Full lines are the NCA simulations, and dashed lines are the results from the phenomenological approach.

positions of the Coulomb peaks show also some deviations, particularly for the IV peak now located at positive $V$. Nevertheless, the intensities and the widths (which are larger than those predicted by the noninteracting resonant-level model) are semiquantitatively reproduced. The small discrepancy in the position of the STM peak at negative $V$ might be due to some dependence with $E_{d}$ of the Haldane shift, being smaller for negative $E_{d}$. Having clarified the physics at play in the asymmetric situation, which simplifies because the system is in equilibrium with a single lead, we now consider the

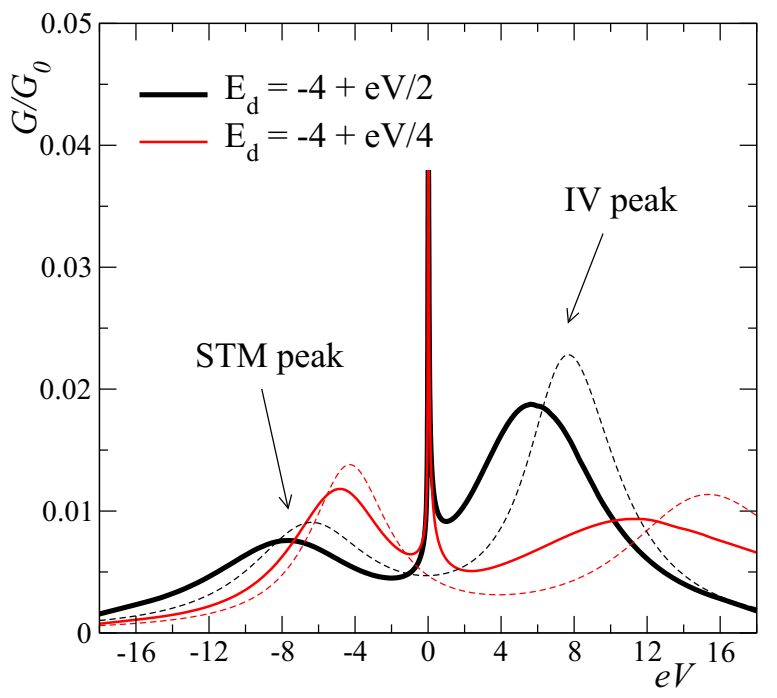

FIG. 5. (Color online) Differential conductance in the localmoment regime $E_{d}^{0}=-4 \Delta=-4$, with the same parameters as in Fig. 4. Full lines are the NCA simulations, and dashed lines are the results from the phenomenological approach. problem in is full complexity for the regime of symmetric tunnel couplings.

\section{Conductance for symmetric tunnel coupling to the leads \\ 1. Numerical NCA results}

For symmetric tunnel coupling to the leads, an approximation like Eq. (4), which would permit us to reduce the problem to the calculation of the spectral density at equilibrium, is no longer possible. In Fig. 6 we show the results of the nonequilibrium NCA calculation for symmetric tunnel barriers, $\Delta_{L}=\Delta_{R}=\Delta / 2$, and parameters similar to those of the experiment by Könemann et al. [15], but with negative $E_{d}^{0}$. In contrast to the results of Sec. IV B 1 , the symmetry now imposes that $G(V)=G(-V)$ for $\Delta_{L}=\Delta_{R}$ and $\alpha=1 / 2$.

While one would expect that the noninteracting resonantlevel model is meaningless in this nonequilibrium interacting case, the symmetric coupling to the leads "blurs" in the conductance the asymmetry due to correlation effects. The simplest noninteracting resonant-level model with renormalized parameters is indeed able to reproduce the Coulomb blockade peaks obtained with the NCA (of course the Kondo anomaly is not captured). Except for the small modification of the $\alpha$ value, which is hard to justify, the changes in the other parameters of the model are expected: a width $4.2 \mathrm{meV}$ nearly two times larger than expected $(2.30 \mathrm{meV})$ because of correlation effects, an effective $E_{d}$ shifted upwards due to renormalization effects (the Haldane shift [35] is $1.04 \mathrm{meV}$ for the half bandwidth $D=20 \mathrm{meV}$ used in the figure), and smaller intensity also due to correlation effects (weight proportional to $1-\left\langle n_{-\sigma}\right\rangle$ ). We now turn to a detailed

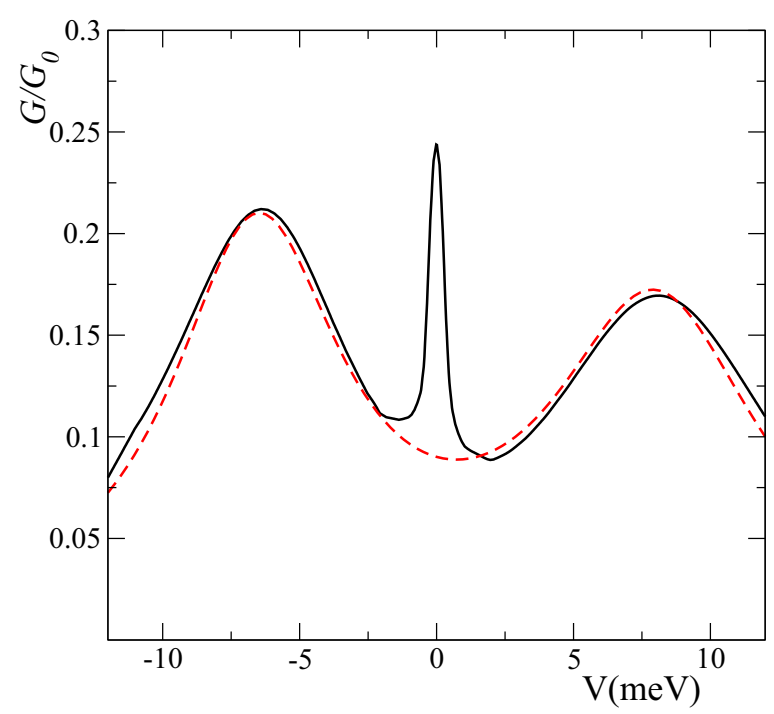

FIG. 6. (Color online) Differential conductance at symmetric tunnel couplings, $\Delta_{L}=\Delta_{R}$, for parameters close to the experimental measurement of Könemann et al. [15], namely $\Delta=1.15 \mathrm{meV}$, $E_{d}^{0}=-4.5 \mathrm{meV}, T=0.014 \mathrm{meV}$, and $\alpha=0.425$. The full line is the nonequilibrium NCA calculation, while the dashed line corresponds to a fit to the noninteracting resonant-level model with renormalized parameters $\Delta^{\text {eff }}=2.1 \mathrm{meV}, E_{d}^{0}=-3.44 \mathrm{meV}, \alpha=0.45$, and a scale reduction factor 0.355 . 
description of the Kondo resonance for a large range of parameters.

\section{On the width of the nonequilibrium Kondo peak}

In this section, we study the relation between the width of the low bias Kondo resonance in the differential conductance $G(V)$ (which is present for negative enough $E_{d}^{0}$ ) and the Kondo peak in the equilibrium spectral density of the impurity level $\rho(\omega)$. We define $T_{K}^{G}$ (resp. $T_{K}^{\rho}$ ) as the energy scale corresponding to half the width at half maximum of the peak in $G(V)$ [resp. $\rho(\omega)$ ] near $V=0$ (resp. $\omega=0$ ). Both quantities are on the order of the Kondo temperature, but differ from a nonuniversal prefactor. Since the Kondo temperature is often extracted experimentally from the differential conductance curve, but theoretically the equilibrium density of states is more standardly computed, for instance with the numerical renormalization group (which has difficulties in grasping the effect of finite bias voltage), we consider here systematically the ratio $T_{K}^{G} / T_{K}^{\rho}$, for several ratios of couplings to the leads $\Delta_{R} / \Delta_{L}$, keeping $\Delta=\Delta_{R}+\Delta_{L}=1$ fixed, and also for several values of the level position $E_{d}$. In this section we keep $E_{d}$ fixed and assume a symmetric voltage drop $\mu_{L}=-\mu_{R}=e V / 2$, as $V$ is increased, and take the half bandwidth $D=10$. Both the differential conductance $G(V)$ and equilibrium density of states $\rho(\omega)$ are computed with the $\mathrm{NCA}$, the former with the nonequilibrium formalism, and the latter at equilibrium $[20,21,36]$. The resulting ratio of Kondo temperatures is shown in Table I.

It can be noticed from Table I that the ratio $T_{K}^{G} / T_{K}^{\rho} \geqslant 1$ and decreases towards unity when both the tunnel asymmetry ratio $R=\Delta_{R} / \Delta_{L}$ is increased and the impurity level position $E_{d}$ is decreased negatively, which can be understood as follows. For large asymmetry of the tunnel coupling to the leads, $R \gg 1$, the dot can be considered at equilibrium with the right lead, and Eq. (4) becomes valid. Furthermore, in the Kondo limit for fixed chemical potential $\mu$, the Kondo peak is insensitive to a small change in the localized level $E_{d}$, and remains close to the Fermi level. For $|e V| \ll T_{K}^{\rho}$ this is supported by Fermi-liquid approaches [40,41]. Thus, one can drop the second term in Eq. (5) and $G(V)$ for $|e V|<T_{K}^{\rho}$ gives directly the spectral density of the dot state $\rho_{R}\left(e V, E_{d}^{0}\right)$. This effect is evident in Fig. 7, where the evolution of the Kondo peak in conductance $G(V)$ with increasing asymmetry ratio $R$ is shown and compared to the equilibrium density of states $\rho_{R}\left(e V, E_{d}^{0}\right)$.

TABLE I. Ratio $T_{K}^{G} / T_{K}^{\rho}$ of the Kondo temperatures extracted from finite bias conductance $G(V)$ and equilibrium density of states $\rho(\omega)$, obtained for different values of the level energy $E_{d}$ and various asymmetry ratios $\Delta_{R} / \Delta_{L}$.

\begin{tabular}{lccccc}
\hline \hline$\Delta_{R} / \Delta_{L} E_{d}$ & -2 & -3 & -4 & -5 & -6 \\
\hline 1 & 1.59 & 1.45 & 1.33 & 1.31 & 1.30 \\
2 & 1.50 & 1.19 & 1.078 & 1.09 & 1.08 \\
5 & 1.29 & 1.03 & 1.008 & 1.003 & $\sim 1$ \\
10 & 1.29 & $\sim 1$ & $\sim 1$ & $\sim 1$ & $\sim 1$ \\
20 & 1.29 & $\sim 1$ & $\sim 1$ & $\sim 1$ & $\sim 1$ \\
30 & 1.29 & $\sim 1$ & $\sim 1$ & $\sim 1$ & $\sim 1$ \\
\hline \hline
\end{tabular}

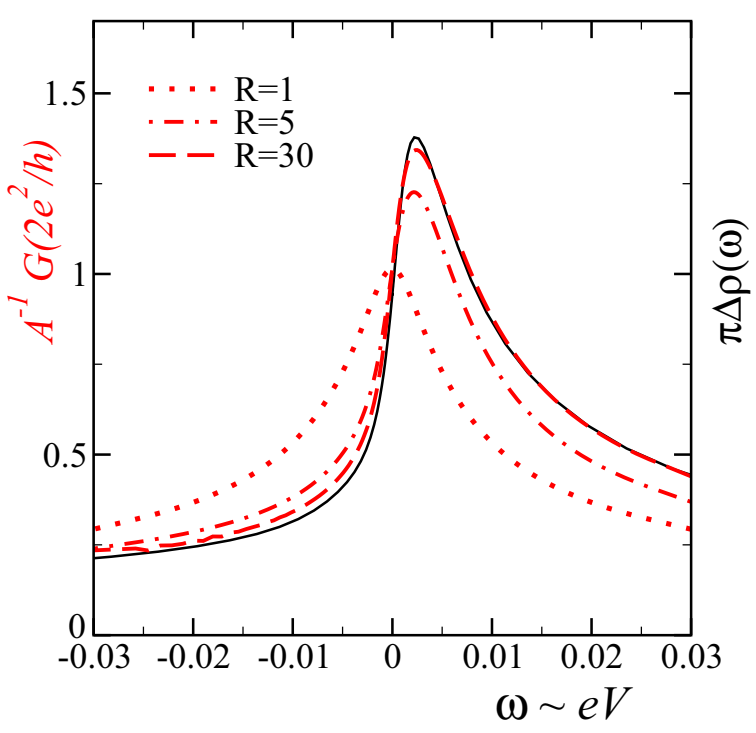

FIG. 7. (Color online) Differential conductance near $G(V)$ (broken lines in red, corresponding to the left vertical scale), and equilibrium spectral density of the quantum dot $\rho(\omega)$ (full line in black, corresponding to the right vertical scale), computed for $\Delta=1$, $E_{d}=-4$, and several tunnel asymmetry ratios $R=\Delta_{R} / \Delta_{L}=$ $1,5,30$. The coefficient $A=4 \Delta_{R} \Delta_{L} / \Delta^{2}$ is introduced to normalize the conductance to the density of states.

\section{COMPARISON WITH EXPERIMENT}

In this last section, we discuss the experiment of Könemann et al. [15]. This experiment corresponds to a value of $E_{d}^{0}$ that is positive and very large (two orders of magnitude bigger than $\Delta$ ). This situation is very hard for the numerical solution of the self-consistent NCA equations, but since the Kondo effect does not take place, we can compare the experiment to the phenomenological approach developed in Sec. IV B 2, which takes into account the changes in both width and height of the charge-transfer peak in the spectral density with the dot level $E_{d}$. The experiment was done for highly asymmetric tunnel coupling to the leads, so that the main assumption of Sec. IV B 2 is fulfilled.

In the experiment, there is a peak at $V=-9.7 \mathrm{mV}$ with a half-width of $76 \mu \mathrm{V}$ and another one at $V=8.9 \mathrm{mV}$ with a half-width of $35.5 \mu \mathrm{V}$ which is about five times more intense. For $\alpha \simeq 1 / 2$ (as suggested in Ref. [15]), the noninteracting resonant level model incorrectly predicts two peaks of equal width and height at opposite voltages (see the end of Sec. III). For $\alpha=1 / 2$, our more accurate phenomenological theory predicts a ratio of about $3 / 2$ between the width of the IV peak at negative $V$ and the STM peak at positive $V$, and not a ratio of about 2 . However, the fact that the positions of the two peaks are not symmetric with respect to zero bias shows that $\alpha$ is in fact different from $1 / 2$. Using $\alpha=0.45$ to adjust the correct peak position, this provides an additional contribution to the ratio in the peak widths. In Fig. 8 we display our results for parameters close to the experiment. While we are unable to reproduce exactly the intensities, positions, and widths of both peaks, we obtain a semiquantitative agreement with experiment. Our conductance curves can be fitted with the sum of two Lorentzians: one located at $V=-9.97 \mathrm{mV}$ 


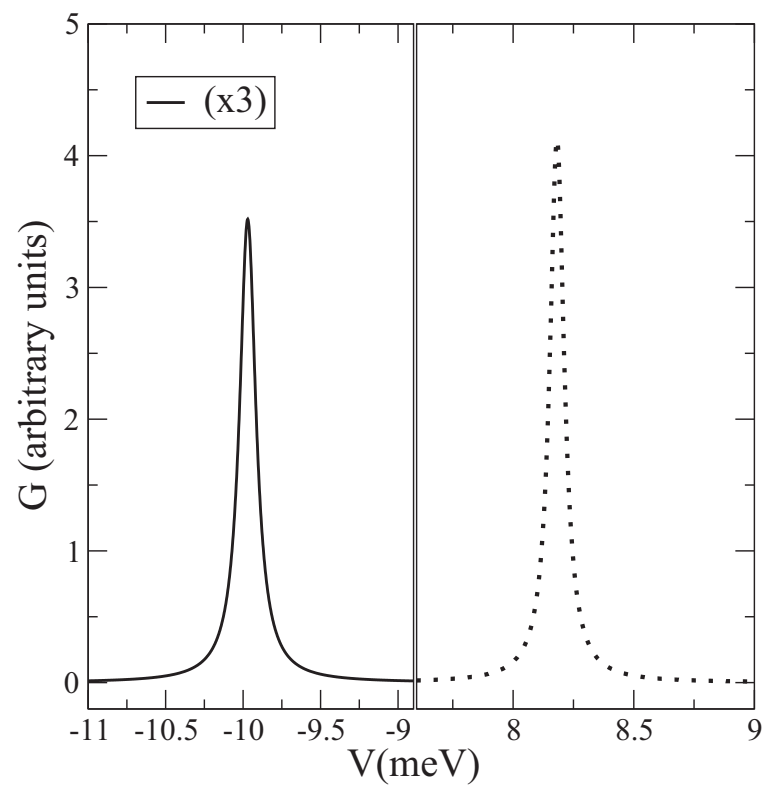

FIG. 8. Differential conductance using the phenomenological model Eq. (11) for parameters corresponding to the experiment by Könemann et al. [15]: $\Delta=0.02 \mathrm{meV}, E_{d}^{0}=4.5 \mathrm{meV}, T=0.01 \mathrm{meV}$, and $\alpha=0.45$. Full (dotted) line corresponds to negative (positive) bias voltage.

with half-width at half maximum $64 \mu \mathrm{V}$ and peak value $1.17 \mathrm{~A}$, another one located at $V=8.18 \mathrm{mV}$, with half-width at half maximum $36 \mu \mathrm{V}$ and peak value $4.21 A$. In the future, comparisons to further experimental data for molecular quantum dots deep in the Kondo regime would be interesting, in order to test our predictions.

\section{SUMMARY}

Motivated by experiments with semiconducting and molecular quantum dots $[15,16]$, we have analyzed the complete shape of the differential conductance $G(V)$ in the infinite- $U$ Anderson model connected to two leads, as a function of the ratio of the potential drops between the dot and both leads, and for various tunneling asymmetries. The simplest situation is the one found in scanning tunneling spectroscopies, where both the potential drop and tunneling rates are strongly asymmetric towards the metallic surface on which a molecule is deposited. As is well known in this situation, the conductance provides exactly a measure of the equilibrium spectral density of the quantum dot and displays only one Coulomb peak when voltage is aligned to the charge-transfer peak in the spectral density (this peak position may contain renormalization effects). When the impurity level is deeply negative, a local moment forms, and the Kondo effect leads to an additional anomaly near zero bias, both in the density of states and differential conductance.

The situation of an arbitrary ratio for the capacitance to the source and drain is more complex (even still assuming very asymmetric tunneling rates). We find that the Coulomb peak position and width in this regime not only display trivial changes expected from the modification of the capacitance ratio, but are also corrected by many-body effects. As is known from previous calculations $[18,19]$ and reproduced by our NCA results, the width of the charge-transfer peak is about two times larger for large and negative level position (local-moment regime) than for large and positive position (empty-orbital regime). In addition, an extra Coulomb peak appears at opposite voltage values to the peak found in the STM situation, as expected from Coulomb blockade theory. However, this peak has in general a different nature, and relates to the intermediate-valence situation of the underlying quantum impurity model, and displays accordingly a width renormalization of about $3 / 2$ compared to expectations based on a noninteracting effective resonant-level model. All these effects can be taken into account by a simple phenomenological approach described in Sec. IV B 2, which is able to describe well our numerical simulations (except for the presence of the Kondo resonance) and agrees semiquantitatively with some published experimental results (see Sec. V).

We also provided a general study of the Kondo temperature extracted from the differential conductance (as typically done in experiments), and made some precise connections to the Kondo scale extracted theoretically from the equilibrium spectral density. We find that the former scale can be up to $60 \%$ larger than the latter one, and that they coincide only for asymmetric tunnel couplings deeply in the Kondo regime.

We hope that all these results will serve as guidelines for interpreting more quantitatively experimental data obtained from molecular quantum dots, since many-body effects can quantitatively alter the predictions of basic Coulomb blockade theory.

\section{ACKNOWLEDGMENTS}

We are partially supported by CONICET, Argentina. This work was sponsored by PICT 2010-1060 and 2013-1045 of the ANPCyT-Argentina, PIP 112-201101-00832 of CONICET, and PICS CNRS-CONICET 5755.
[1] D. Goldhaber-Gordon, H. Shtrikman, D. Mahalu, D. AbuschMagder, U. Meirav, and M. A. Kastner, Nature (London) 391, 156 (1998).

[2] S. M. Cronenwett, T. H. Oosterkamp, and L. P. Kouwenhoven, Science 281, 540 (1998).

[3] D. Goldhaber-Gordon, J. Göres, M. A. Kastner, H. Shtrikman, D. Mahalu, and U. Meirav, Phys. Rev. Lett. 81, 5225 (1998).
[4] W. G. van der Wiel, S. De Franceschi, T. Fujisawa, J. M. Elzerman, S. Tarucha, and L. P. Kouwenhoven, Science 289, 2105 (2000).

[5] W. Liang, M. P. Shores, M. Bockrath, J. R. Long, and H. Park, Nature (London) 417, 725 (2002).

[6] J. J. Parks, A. R. Champagne, G. R. Hutchison, S. Flores-Torres, H. D. Abruña, and D. C. Ralph, Phys. Rev. Lett. 99, 026601 (2007). 
[7] J. J. Parks, A. R. Champagne, T. A. Costi, W. W. Shum, A. N. Pasupathy, E. Neuscamman, S. Flores-Torres, P. S. Cornaglia, A. A. Aligia, C. A. Balseiro, G. K.-L. Chan, H. D. Abruña, and D. C. Ralph, Science 328, 1370 (2010).

[8] G. D. Scott and D. Natelson, ACS Nano 4, 3560 (2010).

[9] S. Florens, A. Freyn, N. Roch, W. Wernsdorfer, F. Balestro, P. Roura-Bas, and A. A. Aligia, J. Phys. Condens. Matter 23, 243202 (2011).

[10] M. Urdampilleta, S. Klyatskaya, J.-P. Cleuziou, M. Ruben, and W. Wernsdorfer, Nat. Mater. 10, 502 (2011).

[11] R. Vincent, S. Klyatskaya, M. Ruben, W. Wernsdorfer, and F. Balestro, Nature (London) 488, 357 (2012).

[12] M. Misiorny, E. Burzurí, R. Gaudenzi, K. Park, M. Leijnse, M. R. Wegewijs, J. Paaske, A. Cornia, and H. S. J. van der Zant, Phys. Rev. B 91, 035442 (2015).

[13] A. C. Hewson, The Kondo Problem to Heavy Fermions (Cambridge University Press, Cambridge, 1993).

[14] M. Pustilnik and L. Glazman, J. Phys. Condens. Matter 16, R513 (2004).

[15] J. Könemann, B. Kubala, J. König, and R. J. Haug, Phys. Rev. B 73, 033313 (2006).

[16] J. Park, Ph.D. thesis, University of California, 2003.

[17] J. Paaske, A. Rosch, P. Woelfle, N. Mason, C. M. Marcus, and J. Nygard, Nat. Phys. 2, 460 (2006).

[18] Th. Pruschke and N. Grewe, Z. Phys. B 74, 439 (1989).

[19] D. E. Logan, M. P. Eastwood, and M. A. Tusch, J. Phys. Condens. Matter 10, 2673 (1998).

[20] N. S. Wingreen and Y. Meir, Phys. Rev. B 49, 11040 (1994).

[21] M. H. Hettler, J. Kroha, and S. Hershfield, Phys. Rev. B 58, 5649 (1998).

[22] P. Roura-Bas, Phys. Rev. B 81, 155327 (2010).

[23] G. C. Tettamanzi, J. Verduijn, G. P. Lansbergen, M. Blaauboer, M. J. Calderón, R. Aguado, and S. Rogge, Phys. Rev. Lett. 108, 046803 (2012).
[24] L. Tosi, P. Roura-Bas, and A. A. Aligia, Phys. Rev. B 88, 235427 (2013).

[25] A. Glatz and I. S. Beloborodov, Phys. Rev. B 81, 033408 (2010).

[26] N. M. Chtchelkatchev, A. Glatz, and I. S. Beloborodov, J. Phys.: Condens. Matter 25, 185301 (2013).

[27] A. A. Aligia, Phys. Rev. B 89, 125405 (2014).

[28] H. Park, J. Park, A. K. L. Lim, E. H. Anderson, A. P. Alivisatos, and P. L. McEuen, Nature (London) 407, 57 (2000).

[29] N. B. Zhitenev, H. Meng, and Z. Bao, Phys. Rev. Lett. 88, 226801 (2002).

[30] M. Galperin, M. A. Ratner, and A. Nitzan, J. Phys.: Condens. Matter 19, 103201 (2007).

[31] S. Ballmann, R. Härtle, P. B. Coto, M. Elbing, M. Mayor, M. R. Bryce, M. Thoss, and H. B. Weber, Phys. Rev. Lett. 109, 056801 (2012); D. Rakhmilevitch, R. Korytár, A. Bagrets, F. Evers, and O. Tal, ibid. 113, 236603 (2014).

[32] P. Roura-Bas, L. Tosi, and A. A. Aligia, Phys. Rev. B 87, 195136 (2013).

[33] Y. Meir and N. S. Wingreen, Phys. Rev. Lett. 68, 2512 (1992).

[34] In the intermediate valence regime and for small temperatures, the width of the charge-transfer peak in the spectral density becomes about two times narrower than expected in the NCA calculations. Therefore we have taken $T \geqslant \Delta$ to calculate correctly its width in this regime.

[35] F. D. M. Haldane, Phys. Rev. Lett. 40, 416 (1978).

[36] L. Tosi, P. Roura-Bas, and A. A. Aligia, J. Phys. Condens. Matter 24, 365301 (2012).

[37] H. J. Leder and G. Czycholl, Z. Phys. B. 35, 7 (1979).

[38] A. A. Aligia and B. Alascio, J. Magn. Magn. Matter 46, 321 (1985).

[39] J. Hubbard, Proc. R. Soc. A281, 401 (1964).

[40] E. Sela and J. Malecki, Phys. Rev. B 80, 233103 (2009).

[41] A. A. Aligia, J. Phys.: Condens. Matter 24, 015306 (2012); Phys. Rev. Lett. 111, 089701 (2013). 